\title{
Estudio del comportamiento fisiológico de la semilla de tres especies cultivadas de Passiflora L. (Passifloraceae) como una contribución para la conservación ex situ
}

\section{Study of the physiological behavior of the seeds of three cultivated species of Passiflora L. (Passifloraceae) for contribution to ex situ conservation}
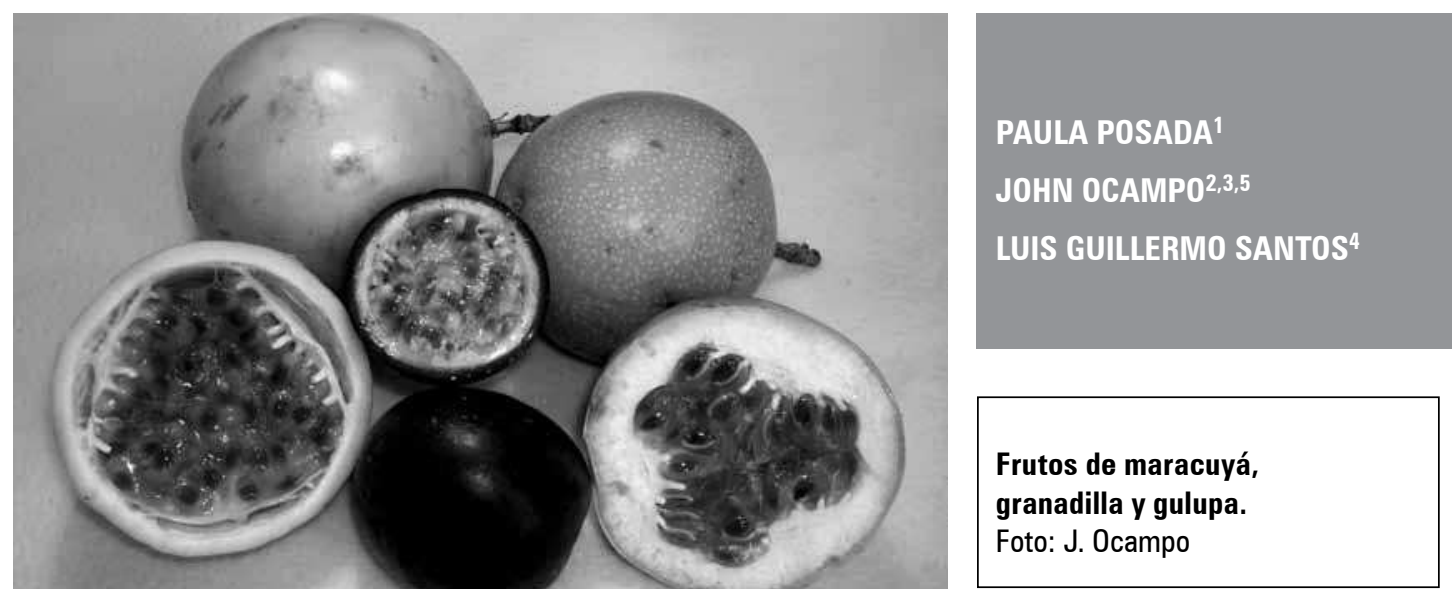

\section{RESUMEN}

El maracuyá ( $P$. edulis f. flavicarpa), la granadilla ( $P$. ligularis) y la gulupa ( $P$. edulis f. edulis) son económicamente las principales especies del género Passiflora L. por su fruto comestible y comercialización en mercados locales e internacionales. El objetivo de este estudio fue identificar el comportamiento fisiológico de la semilla de maracuyá, granadilla y gulupa frente al almacenamiento como una contribución a la conservación ex situ. El diseño experimental utilizado fue completamente al azar con cinco tratamientos y tres repeticiones siguiendo el protocolo de Hong y Ellis (1996). Las semillas de maracuyá y gulupa fueron secadas en cuartos con flujo continuo de aire $\left(20^{\circ} \mathrm{C} \pm 2\right.$ y $35 \%$ humedad relativa [HR]), donde se les disminuyó el contenido de humedad hasta un rango óptimo de 10-12\% en 5 horas y obteniendo una germinación de 100 y 77,1\%, respectivamente. En la granadilla, la desecación tardó 20 horas y las semillas germinaron en un $79,5 \%$. Posteriormente, la disminución hasta $6 \%\left(20^{\circ} \mathrm{C} \pm 2\right.$ y $\left.10 \% \mathrm{HR}\right)$ tardó 60 horas, para maracuyá y gulupa con una germinación de 81,2 y $98,6 \%$, respectivamente y 105 horas para granadilla con un $91,4 \%$

Facultad de Ciencias Agropecuarias, Programa de Maestría en Ciencias Biológicas, Universidad Nacional de Colombia, Palmira (Colombia).

Facultad de Ciencias Agropecuarias, Universidad Nacional de Colombia, Palmira (Colombia).

Decision and Policy Analysis Program (DAPA), Centro Internacional de Agricultura Tropical (CIAT), Palmira (Colombia).

Programa de Recursos Genéticos, Centro Internacional de Agricultura Tropical (CIAT), Palmira (Colombia).

Autor para correspondencia. jaocampop@unal.edu.co 
de germinación. De acuerdo con el protocolo mencionado, se conservaron durante 6 meses, evaluándose en el primero, tercero y sexto mes con una germinación promedio del $94,1 \%$ para maracuyá, 90,9\% para granadilla y $87,9 \%$ para gulupa. Lo anterior sugiere que las semillas de las tres especies estudiadas presentan tolerancia a la desecación y posiblemente pertenecen a la categoría ortodoxa. Estos resultados contribuyen al manejo y conservación de los recursos genéticos de los frutales neotropicales.

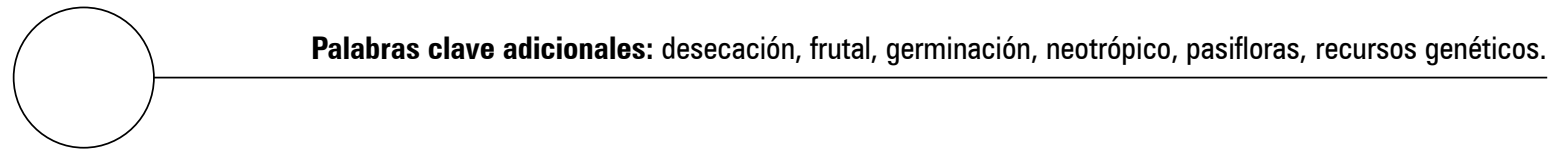

\section{ABSTRACT}

Yellow (P. edulis f. flavicarpa), sweet (P. ligularis) and purple passion fruits (P. edulis f. edulis) are economically the main species of the Passiflora L. genus with edible fruits that are marketed in local and international markets. The objective of this study was to identify the physiological behavior of the seeds of yellow passion fruit, sweet passion fruit and purple passion fruit during storage for contribution to ex situ conservation. The experimental design was completely randomized with five treatments and three replications following the protocol of Hong and Ellis (1996). Firstly, the seeds were dried in chambers with a continuous air flow $\left(22^{\circ} \mathrm{C}\right.$ and $35 \%$ relative humidity [RH]), with a decrease in moisture content to the optimum range of $10-12 \%$, for 5 hours for yellow passion fruit and purple passion fruit, obtaining $100 \%$ and $77.1 \%$ germination, respectively. In the case of sweet passion fruit, desiccation took 20 hours with $79.5 \%$ germination. Afterwards, decreasing the moisture content to $6 \%\left(21^{\circ} \mathrm{C}\right.$ and $\left.10 \% \mathrm{RH}\right)$ took 60 hours for yellow passion fruit and purple passion fruit, with a germination of $81.2 \%$ and $98.6 \%$, respectively, and 105 hours for sweet passion fruit, with $91.4 \%$ germination. According to the mentioned protocol, the treatments underwent conservation assessments for 6 months at the $1^{\text {st }}, 3^{\text {rd }}$ and $6^{\text {th }}$ month, with an average germination of $94.1 \%$ for yellow passion fruit, $90.9 \%$ for sweet passion fruit and $87.9 \%$ for purple passion fruit. This suggests that the seeds of the three species are tolerant to desiccation and possibly belong to the orthodox category. These results contribute to the management and conservation of plant genetic resources of Neotropical fruit.

Additional key words: desiccation, fruit, germination, Neotropical, passion fruit, genetic resources.

Colombia es el país con mayor diversidad de Passifloraceae con 170 especies, tanto en formas silvestres como cultivadas (Ocampo, 2007). El maracuyá (Passiflora edulis f. flavicarpa Degener), la granadilla (P. ligularis Juss.) y la gulupa (P. edulis f. edulis Sims) son las especies más importantes del género Passiflora L. por su fruto comestible y la comercialización en mercados nacionales e internacionales. Su polinización es cruzada (alogamia) y realizada principalmen- te por insectos de Xylocopa spp., los cuales son atraídos por el dulce del néctar de la flor (Rendón et al., 2013; Arias et al., 2014). Estas especies son originarias del trópico suramericano e introducidas a otras latitudes como México, Kenia, India, Tailandia, Australia y Nueva Zelanda como planta ornamental y en cultivos comerciales. Colombia cuenta con aproximadamente 11.000 ha cultivadas con estas especies, siendo Huila, Valle del Cauca, Cundinamarca, Boyacá 
y Antioquia los departamentos en donde se concentra la producción (Parra et al., 2013). A pesar de este potencial, existe poca información sobre el comportamiento fisiológico de la semilla durante el almacenamiento de las especies cultivadas de Passiflora y la mayoría de los estudios se han concentrado en sus propiedades para la alimentación y uso en la farmacopea.

La conservación ex situ de los principales cultivos de seguridad alimentaria global es una de las prioridades de la humanidad desde la revolución neolítica y actualmente se conservan accesiones de 156 cultivos en bancos de germoplasma en diferentes países (Westengen et al., 2013). Por otro lado, la conservación de los recursos genéticos de los frutales tropicales ha sido poco implementada y en especial la ex situ en bancos de germoplasma en los países andinos (Lobo, 2006). La longevidad de las semillas varía de manera importante tanto intra como interespecíficamente debido a las diferencias de genotipo y origen (Hong y Ellis, 1996). Actualmente, se reconocen tres categorías principales del comportamiento fisiológico de las semillas en almacenamiento: ortodoxa, intermedia y recalcitrante (Hong y Ellis, 1996). Estas categorías se basan en la longevidad y la capacidad de germinación de las semillas almacenadas más allá del término prolongado en condiciones ambientales apropiadas. Asimismo, el mantenimiento de la viabilidad de semillas con comportamiento intermedio o recalcitrante es problemático para la conservación ex situ y por esta razón el 90\% de las accesiones en los bancos de germoplasma preservan semillas ortodoxas (Rao et al., 2007).

Por su parte, las investigaciones en Passiflora spp. relacionadas con el comportamiento fisiológico de la semilla se han enfocado en los tratamientos pregerminativos a diferentes temperaturas y con concentraciones variables de ácido giberélico $\left(\mathrm{AG}_{3}\right)$ o la escarificación en condiciones de laboratorio y vivero. Así, la aplicación de $\mathrm{AG}_{3}$ a una concentración entre 300 a $1000 \mathrm{mg} \mathrm{L}^{-1}$ en semillas de $P$. tripartita var. mollissima (Cardozo,
1998), P. nítida (Passos et al., 2004), P. alata (Ferreira et al., 2005; Rosseto et al., 2000), P. edulis f. flavicarpa (Lima et al., 2009) y P. ligularis (Cárdenas, 2011) promovió considerablemente la germinación. En adición, la escarificación y la inmersión de la semilla $\left(50^{\circ} \mathrm{C}\right.$ por $\left.5 \mathrm{~min}\right)$ de $P$. tripartita var. mollissima (Ramírez et al., 2008), P. tricuspis (Delanoy et al. 2006), P. caerualea (Mendiondo y García, 2009), P. cincinnata (Júnior et al., 2009), P. ligularis, $P$. maliformis y $P$. edulis $\mathrm{f}$. edulis (Gutiérrez et al., 2011) pueden favorecer la absorción del agua y romper la latencia. En otro estudio, se estableció que la temperatura alternada de 20 a $30^{\circ} \mathrm{C}$ disminuye la dormancia de las semillas en $P$. alata (Osipi y Nakagawa, 2005). Del mismo modo, Balaguera et al. (2010) con la estratificación en frío $\left(4^{\circ} \mathrm{C}\right)$ y cubrimiento de la semilla de P. edulis f. edulis, obtuvieron los mayores porcentajes de germinación $>80 \%$.

En cuanto al almacenamiento, Nakagawa et al. (1991) determinaron que la semilla de maracuyá conservada en cuarto frío $\left(4^{\circ} \mathrm{C}\right)$ durante 57 meses, disminuye el porcentaje de germinación al $60 \%$. Por el contrario, cuando la semilla se preservó a $10^{\circ} \mathrm{C}$ con un contenido de humedad de $12 \%$ en un periodo de 12 meses, la germinación alcanza el 85\% (Fonseca y Silva, 2005). Asimismo, la viabilidad de la semilla de maracuyá cuando es secada al 10\% y preservada en empaques herméticos durante un periodo de 4 meses no presenta pérdidas significativas y sugieren un aparente comportamiento ortodoxo (Torres y Ellis, 2007). En otra investigación en la misma especie, Carlesso et al. (2008) mencionan que las semillas secadas a la sombra en temperaturas de 30 a $40^{\circ} \mathrm{C}$ y almacenadas a $15^{\circ} \mathrm{C}$ durante seis meses afectan su calidad fisiológica. En otras especies, como $P$. alata y $P$. setacea la conservación de semillas a $10^{\circ} \mathrm{C} \mathrm{du-}$ rante un tiempo hasta de 4 meses es más eficaz, mientras que en $P$. cincinnata puede conservarse por un periodo superior. Por otro lado, la técnica de criopreservación de semillas en nitrógeno líquido $\left(-196^{\circ} \mathrm{C}\right)$ ha demostrado su éxito en la conservación de la viabilidad de 22 especies de Passiflora, que presentaron un comportamiento intermedio 
y ortodoxo (Hong et al., 1998; Liu et al., 2008; Veiga-Barbosa et al., 2013). Sin embargo, en P. edulis $\mathrm{f}$. flavicarpa, $P$. ligularis, $P$. alata, $P$. cincinnata, $P$. foetida, y $P$. tripartita var. mollissima existen discrepancias entre diferentes autores, respecto al tipo de semilla frente al almacenamiento (Teng, 1977; Becwar et al., 1983; Ospina et al., 2000; Guevara et al., 2003; González-Benito et al., 2009). Además, en ninguna de estas investigaciones anteriores menciona el uso del protocolo de Hong y Ellis (1996) para determinar el comportamiento de las semillas en almacenamiento, el cual ha sido desarrollado y probado en numerosas especies vegetales. Por tales razones, el objetivo de este estudio fue determinar el comportamiento fisiológico de la semilla del maracuyá, la granadilla y la gulupa frente al almacenamiento como un aporte para la conservación ex situ.

\section{MATERIALES Y MÉTODOS}

Esta investigación fue realizada en los laboratorios del Programa de Recursos Genéticos del Centro Internacional de Agricultura Tropical (CIAT), localizado en el municipio de Palmira (Valle del Cauca, Colombia) a una altitud de 1.000 msnm.

Se utilizaron frutos maduros cosechados de plantas de 15 meses de edad provenientes de las colecciones nacionales de maracuyá, granadilla y gulupa establecidas en los municipios de Palestina
(1.180 msnm) y Manizales (2.280 msnm) en el departamento de Caldas (Colombia). Los frutos cosechados fueron lavados con agua a temperatura ambiente para eliminar cualquier riesgo de contaminación de las semillas. La pulpa con las semillas se extrajo manualmente y el arilo fue removido con la ayuda de la enzima Citrozym ${ }^{\circledR}$ Ultra L, a una concentración de $1 \mathrm{~mL} 500 \mathrm{~mL}^{-1}$ de agua por cada $5 \mathrm{~kg}$ de pulpa, y agitando manualmente cada 30 min durante $2 \mathrm{~h}$. Posteriormente, las semillas fueron lavadas y secadas sobre toallas de papel absorbente por un periodo de $10 \mathrm{~min}$ a temperatura ambiente. Finalmente, se tomaron $5 \mathrm{~g}$ de semillas que se trituraron con un molino eléctrico (Cuisinart DCG-20N ${ }^{\circledR}$ ). Estas semillas, fueron separadas en tres muestras de $1 \mathrm{~g}$ cada una y empleadas para determinar el porcentaje de humedad con el uso de una termobalanza MB45 $\left(\mathrm{OHAUS}^{\circledR}\right)$.

Una prueba de germinación se realizó en un sustrato de arena previamente tratada con vapor a $121^{\circ} \mathrm{C}$ durante $4 \mathrm{~h}$, para determinar la viabilidad de las semillas en fresco mediante la obtención del porcentaje (figura 1). La evaluación de la germinación se llevó a cabo cada 48 h durante 30 d siguiendo la metodología ISTA (2009) y el índice de velocidad de germinación (IVG) fue determinado siguiendo la fórmula de Macguire (1962):

IVG $=\frac{\text { Número de semillas germinadas }}{\text { Tiempo de germinación }}$



Figura 1. Apariencia de las semillas sin arilo de maracuyá (a), granadilla (b) y gulupa (c). 
Acondicionamiento y conservación de la semilla

El comportamiento fisiológico de semilla se estableció siguiendo el protocolo de Hong y Ellis (1996), el cual se basa en la disminución del contenido de humedad y la temperatura con posteriores pruebas de viabilidad (ISTA, 2009). El secado de la semilla o desecación se realizó en cuartos de secado a una temperatura de $20^{\circ} \mathrm{C} \pm 2$, con condiciones variables de 30 y $10 \%$ HR (humedad relativa) y tiempo. Adicionalmente, los tratamientos para este ensayo fueron establecidos de manera progresiva de acuerdo con los resultados de cada uno de ellos:

Tratamiento 1, secado de la semilla hasta alcanzar un contenido de humedad de 10 a 12\%, y validado con una prueba de germinación.

Tratamiento 2, secado de la semilla hasta alcanzar un contenido de humedad de 5 a $6 \%$, y validado con una prueba de germinación.

Tratamiento 3, una muestra de $100 \mathrm{~g}$ de semillas del tratamiento anterior con contenido de humedad de 5 a $6 \%$, empacadas en bolsas de aluminio plástico al vacío (Fuji Impulse Co. V300, Deerfield, IL), conservadas a $-20^{\circ} \mathrm{C}$ durante 1 mes y validada con una prueba de germinación.

Tratamiento 4, una muestra de $100 \mathrm{~g}$ de semillas del tratamiento anterior con contenido de humedad de 5 a $6 \%$, empacadas en bolsas de aluminio plástico al vacío, conservadas a $-20^{\circ} \mathrm{C}$ durante 3 meses y validada con una prueba de germinación.

Tratamiento 5, una muestra de $100 \mathrm{~g}$ de semillas del tratamiento anterior con contenido de humedad de 5 a $6 \%$, empacadas en bolsas de aluminio plástico al vacío, conservadas a $-20^{\circ} \mathrm{C}$ durante 6 meses y validada con una prueba de germinación.

\section{ANÁLISIS DE DATOS}

El diseño experimental empleado fue completamente al azar con cinco tratamientos, tres repeticiones con 70 semillas para cada una y una confiabilidad del 95\%. Los datos fueron tabulados en Microsoft Excel $2010^{\circledR}$ y sometidos al análisis de varianza (Anova) con una previa transformación del porcentaje de germinación con la función Arcoseno (Wagner et al., 2006). Los promedios entre los tratamientos fueron analizados mediante la prueba de comparación de Tukey (HSD) a un nivel de significancia del 5\% y procesados con el programa estadístico SAS v9.

\section{RESULTADOS}

\section{Contenido de humedad y germinación}

Las semillas frescas extraídas de los frutos y libres del arilo presentaron valores promedio de contenido de humedad y porcentajes de germinación variables para las tres especies hasta los 30 d después de la siembra (dds). El maracuyá tuvo germinación del $87 \%$ a un contenido de humedad $(\mathrm{CH})$ de $24,6 \%$, para la granadilla fue de $59 \%$ a un $\mathrm{CH}$ de $34,7 \%$ y para la gulupa la germinación fue de $71 \%$ (CH: $24,0 \%$ ). Por otro lado, el comportamiento de la germinación en las tres especies inició entre los 14 (maracuyá), 16 (granadilla) y 22 (gulupa) dds, respectivamente (figura 2). El mayor índice de velocidad de germinación (IVG) se obtuvo con la semilla de maracuyá un valor de 2, para la granadilla de 1,4 y la gulupa 1,7. Estos últimos resultados indican que las semillas de las especies estudiadas no pueden ser sembradas directamente después la extracción, debido a que un IVG de 2,5 equivale a 100\% de germinación según Macguire (1962).

\section{Acondicionamiento de la semilla}

Tratamiento 1 (contenido de humedad de la semilla del 12\%): las semillas de maracuyá y gulupa, las cuales tenían un contenido de humedad inicial del 24,6 y $24 \%$ respectivamente, tardaron $5 \mathrm{~h}$ de secado en alcanzar el $12 \%$ de humedad en el cuarto de secado a $20^{\circ} \mathrm{C} \pm 2$ y $10 \%$ HR. No obstante, la semilla de granadilla nece- 

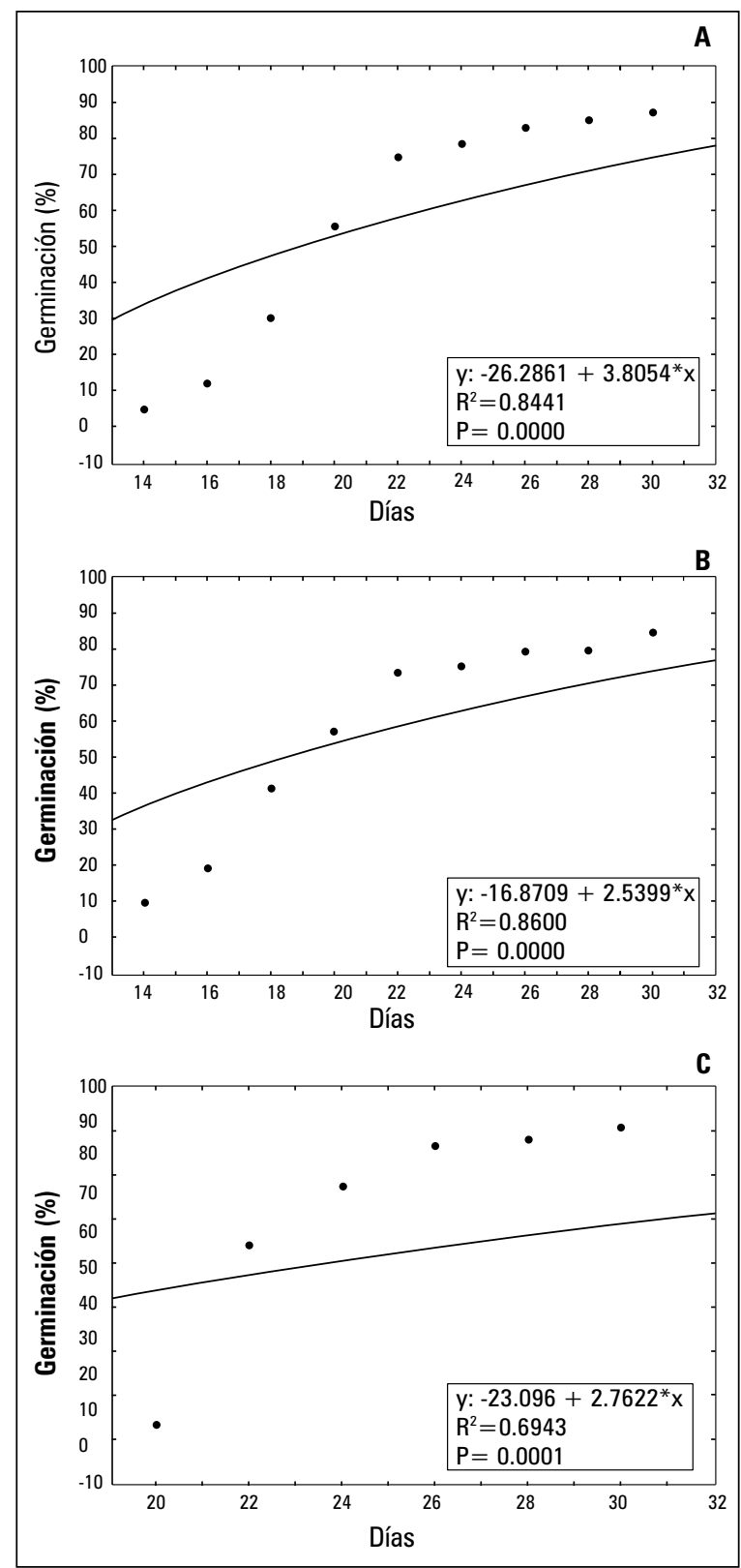

Figura 2. Prueba de germinación previa con las semillas en fresco: (A) maracuyá, (B) granadilla y (C) gulupa. Datos ajustados con tendencia logarítmica.

sitó un total de 20 h de secado, debido al mayor contenido de humedad inicial (34,7\%). Esta última se sometió a dos tipos de secado, el primero en un cuarto a $20^{\circ} \mathrm{C} \pm 2$ y $30 \%$ HR durante 7 h y posteriormente se trasladaron a otro cuarto por $13 \mathrm{~h}$ a $20^{\circ} \mathrm{C} \pm 2$ y $10 \% \mathrm{HR}$, alcanzando un contenido de humedad de $12,44 \%$. Los resultados ob- tenidos de las pruebas de germinación indicaron que la semilla de maracuyá presentó el mayor promedio de germinación con un $100 \%$, seguido de la granadilla y la gulupa con 79,4 a $77,1 \%$, respectivamente (figura 3).

Tratamiento 2 (contenido de humedad de la semilla del 6\%): para este se utilizó una muestra de $100 \mathrm{~g}$ de las mismas semillas del tratamiento 1. En el caso del maracuyá y la gulupa se alcanzó un contenido de humedad de 6,02 y $5,82 \%$ para un periodo de $60 \mathrm{~h}$ en el cuarto a $20^{\circ} \mathrm{C} \pm 2$ y $30 \% \mathrm{HR}$. Por otro lado, la semilla de granadilla alcanzó un contenido de humedad de $5,89 \%$ en 105 h. Los resultados obtenidos en este tratamiento indican que las tres especies presentaron un promedio total de germinación de un $81,2 \%$ para el maracuyá, $91,4 \%$ en granadilla y $98,6 \%$ en gulupa (figura 3 ).

\section{Conservación de la semilla}

Tratamientos 3, 4 y 5 (conservación a $-20^{\circ} \mathrm{C}$ ): las muestras de semillas del tratamiento 2 fueron almacenadas a $-20^{\circ} \mathrm{C}$ por un periodo de 6 meses. Las pruebas de germinación realizadas en el primero, tercero y sexto mes de conservación mostraron que las semillas de las especies estudiadas presentaron porcentajes superiores al 84\% (figura 3). De acuerdo con los resultados obtenidos, la semilla del maracuyá aumenta considerablemente el potencial de germinación con respecto al tiempo de conservación (1 mes: 90\%, 3 meses: 92,3\%, 6 meses: 100\%). En la granadilla el comportamiento de la germinación fue similar (1 mes: 85,3\%, 3 meses: 91,7\%, 6 meses: $95,2 \%$ ), aunque con menor porcentaje en comparación con el maracuyá. En contraste, la semilla de gulupa presentó un comportamiento irregular en el tercer mes, pero con un porcentaje de germinación superior al 84\% (1 mes: 87\%, 3 meses: 84,1\%, 6 meses: 92,8\%).

En cuanto a la prueba de comparación entre promedios de Tukey, mostró que el maracuyá no hubo diferencias significativas $(P \leq 0,05)$ en- 


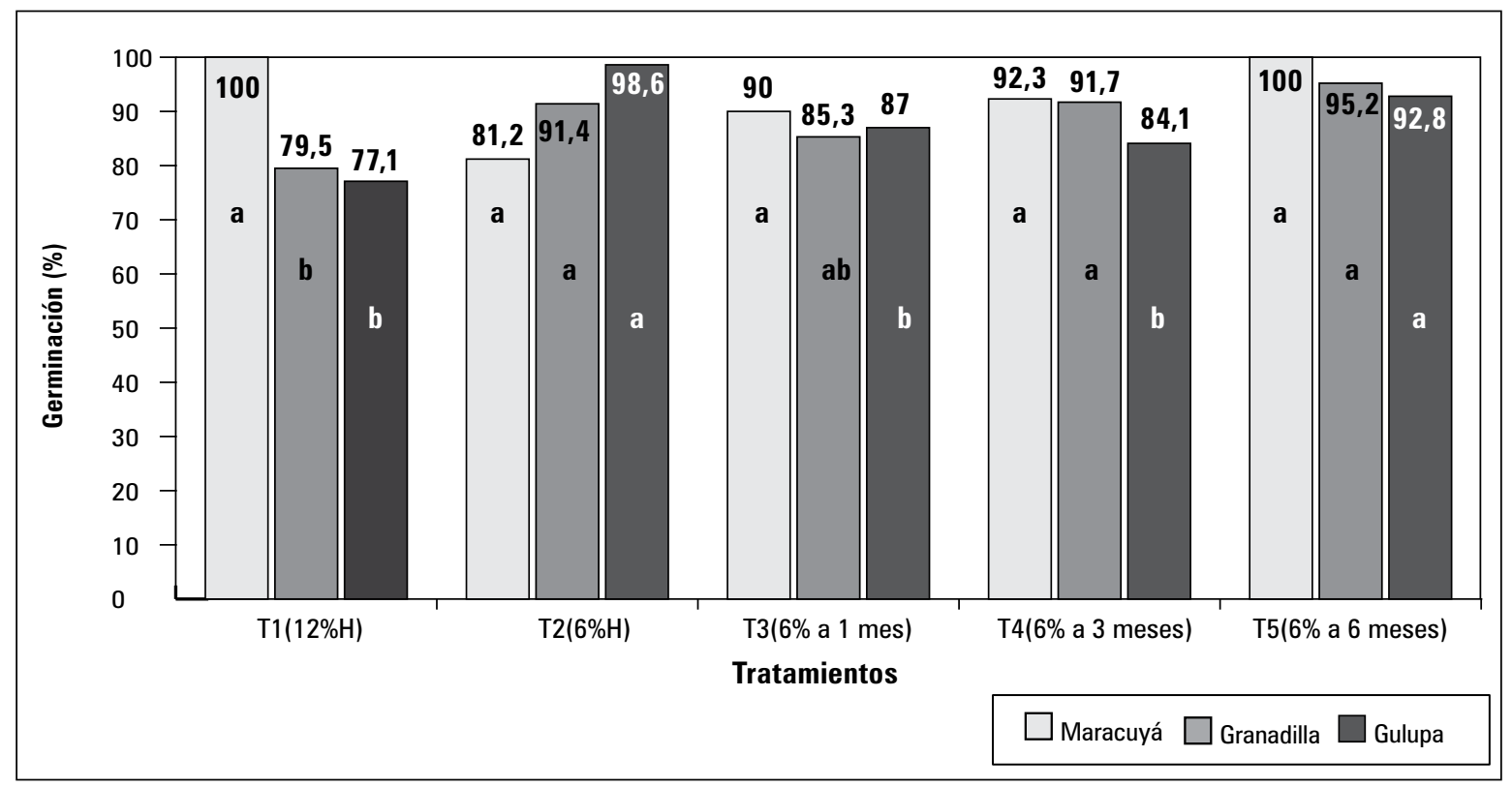

Figura 3. Germinaciones promedio obtenidas para las semillas de maracuyá, granadilla y gulupa, siguiendo el protocolo de Hong y Ellis (1996). Promedios con letras distintas en cada serie indican diferencia significativa según la prueba de Tukey $(P \leq 0,05)$.

tre los tratamientos (figura 3). Sin embargo, la disminución de humedad de la semilla al $6 \%$ y sin conservación en frío $\left(-20^{\circ} \mathrm{C}\right)$ causa un efecto negativo en la germinación (100 a 81,2\%). En la granadilla, las diferencias entre los promedios de los tratamientos T1, T2 y T3 fueron significativas, pero no entre los T2, T4 y T5. Los máximos porcentajes de germinación se presentaron cuando la semilla se secó al $6 \%$ de humedad y se siembra inmediatamente o se conserva durante 6 meses (T2, 91,4\%; T5, 95,2\%). Lo anterior indica que la disminución de la humedad al $6 \%$ en la semilla de la granadilla no afecta la germinación y además si esta es conservada favorece el proceso.

Para la gulupa, los tratamientos T1, T3 y T4 no mostraron diferencias estadísticas $(P \leq 0,05)$, aunque los porcentajes de germinación presentaron valores de 77,1; 87,0 y 84,1\%, respectivamente. Los tratamientos T2 y T5 alcanzaron las mejores germinaciones promedio con valores superiores al 92\%. Esto significa que el comportamiento de la semilla en esta especie es muy variable, puesto que los mayores porcentajes de germinación se presentan cuando el contenido de humedad se disminuye al $6 \%$ y es sembrada inmediatamente o conservada en frío durante 6 meses.

\section{DISCUSIÓN}

Los resultados de las pruebas de germinación concuerdan con lo reportado por Maciel et al. (1997) en maracuyá, ya que se inicia entre los 10 a 15 dds. Asimismo, los porcentajes de germinación de las semillas de las tres especies se equilibraron entre los 22 y 26 dds con valores superiores al $50 \%$ de germinación. Los resultados sugieren que las semillas de las especies estudiadas no presentaron dormancia, debido a que estas germinaron sin necesidad de tratamientos pregerminativos, tal como se ha reportado en otras especies (Manjkhola et al., 2003). Además, las diferencias intra e interespecíficas en los porcentajes y la velocidad de germinación pueden estar asociados al bajo grado de domes- 
ticación presente en las pasifloras cultivadas (Lobo, 2006).

Los resultados de los diferentes tratamientos siguiendo el protocolo de Hong y Ellis (figura 4) sugieren que el almacenamiento en frío promueve el aumento de la germinación de las semillas y concuerdan con los estudios de Manjkhola et al. (2003) y Balaguera et al. (2010) en otras especies. En esta investigación, cuando las semillas fueron conservadas durante 6 meses en frío $\left(-20^{\circ} \mathrm{C}\right)$ con un $6 \%$ de contenido de humedad mostraron un aumento en la germinación y sugiere que este tratamiento puede romper la dormancia física de la semilla.

Por otro lado, las investigaciones reportadas sobre el almacenamiento convencional y la crioconservación en algunas especies de pasifloras difieren en el tipo de comportamiento de la semilla. En el maracuyá, los primeros estudios realizados con el uso de la crioconservación determinaron que

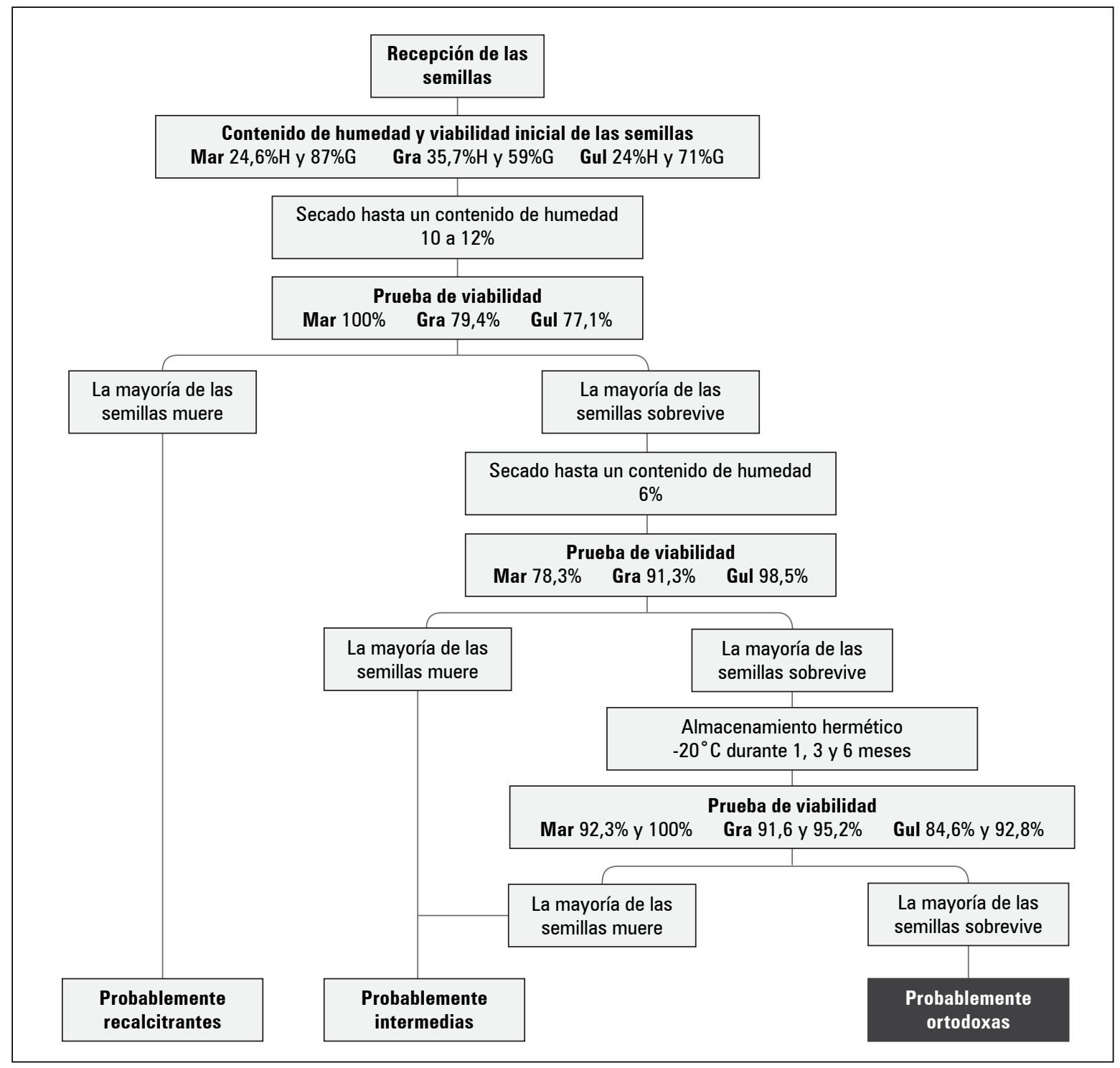

Figura 4. Esquema del protocolo de Hong y Ellis (1996) para las especies estudiadas. Mar: maracuyá; Gra: granadilla; Gul: gulupa. 
la semilla presenta un comportamiento de tipo intermedio de acuerdo con los registros de Teng (1977), Becwar et al. (1983), Ospina et al. (2000) y Guevara et al. (2003). Estos resultados contrastan con los obtenidos en esta investigación y los realizados por González-Benito et al. (2009) y Veiga-Barbosa et al. (2013) en crioconservación, ya que las semillas de maracuyá mostraron un comportamiento ortodoxo al almacenamiento. En adición, en la granadilla Ospina et al. (2000), Guevara et al. (2003) y González-Benito et al. (2009) indican de igual forma que es ortodoxa, aunque Liu et al. (2008) la clasifican como intermedia. En la gulupa, los estudios mencionan que la semilla frente al almacenamiento presenta un comportamiento intermedio cuando es crioconservada (Liu et al., 2008; Veiga-Barbosa et al., 2013). Sin embargo, cuando se siguió el protocolo de Hong y Ellis (1996) en este estudio, la semilla presentó un porcentaje de germinación superior al $85 \%$, lo que sugiere un comportamiento probablemente de tipo ortodoxo.

Finalmente, estos resultados contribuyen al manejo y conservación de los recursos genéticos de las pasifloras. Así mismo, contribuirán a los programas de mejoramiento genético o a la constitución de un banco de germoplasma de estas especies como un aporte a la promoción y uso de los frutales neotropicales con especial énfasis en la región Andina.

\section{CONCLUSIONES}

Los resultados permiten concluir que el comportamiento fisiológico de las semillas de las tres especies estudiadas frente al almacenamiento es posiblemente de tipo ortodoxo y soportan los resultados obtenidos por Torres y Ellis (2007) en maracuyá de acuerdo con el protocolo de Hong y Ellis (1996).

Los mayores porcentajes de germinación de las semillas de maracuyá, granadilla y gulupa se presentaron cuando estas son conservadas a $-20^{\circ} \mathrm{C}$ con una humedad de $6 \%$ durante un periodo de 6 meses (T5) y empacadas en bolsas herméticas de aluminio plástico al vacío. Esto indica que estas son las mejores condiciones para el almacenamiento de las semillas en el presente estudio y el protocolo puede ser adoptado para otras especies de pasifloras con comportamiento ortodoxo, como $P$. alata, $P$. cincinnata y $P$. ambigua.

\section{AGRADECIMIENTOS}

Los autores expresan sus agradecimientos al Ministerio de Agricultura y Desarrollo Rural de Colombia (MADR) y al Centro Internacional de Agricultura Tropical (CIAT, Programa de Recursos Genéticos) por el financiamiento y la logística en el desarrollo de esta investigación.

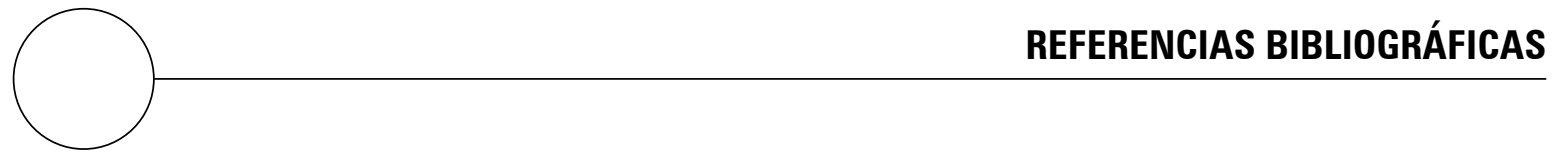

Arias, J.C., J. Ocampo y R. Urrea. 2014. La polinización natural en el maracuyá (Passiflora edulis f. flavicarpa Degener) como un servicio reproductivo y ecosistémico. Agron. Mesoamer. 25(1), 73 83.

Balaguera, H.E., J.G. Álvarez y J. Cárdenas. 2010. Efecto de la estratificación fría y la cobertura plástica en semillas de gulupa (Passiflora edulis Sims.) para la obtención de plántulas. Rev. UDCA Act. Divulg. Cient. 13(2), 89-97.
Becwar, M.R., P.C. Stanwood y K.W. Leonhardt. 1983. Dehydration effects on freezing characteristics and survival in liquid nitrogen of desiccation-tolerant and desiccation sensitive seeds. J. Amer. Soc. Hort. Sci. 108, 613-618.

Cárdenas, J.F. 2011. Morfología y tratamientos pregerminativos de semillas de granadilla (Passiflora ligularis Juss.). Tesis de maestría. Facultad de Agronomía, Universidad Nacional de Colombia, Bogotá. 
Carlesso, V.O., P.A. Berbert, R.F. Silva y E. Detmann. 2008. Secagem e armazenamento de sementes de maracujá amarelo (Passiflora edulis Sims f. flavicarpa Degener). Rev. Bras. Sem. 30(2), 65-74.

Cardozo, H. 1998. Efecto de la escarificación y la dosis del ácido giberélico $\left(\mathrm{AG}_{3}\right)$ en la germinación de semillas de curuba (Passiflora mollisima). Acta Biol. Colomb. 1(4), $127-132$.

Delanoy, M., P. Van Damme, X. Scheldeman y J. Beltran. 2006. Germination of Passiflora mollissima (Kunth) L.H.Bailey, Passiflora tricuspis Mast. and Passiflora nov sp. seeds. Sci. Hortic. 110(2), 198-203.

Ferreira, G., A. Oliveira, J.D. Rodrigues, G.B. Dias, A.M. Detoni, S.M. Tesser y A.M. Antunes. 2005. Efeito de arilo na germinação de sementes de Passiflora alata Curtis em diferentes substratos e submetidas a tratamentos com giberelina. Rev. Bras. Frutic. 27(2), 277-280.

Fonseca, S. y W. Silva. 2005. Conservação de sementes de maraujá amarelo: interferencias do teor de agua das sementes e da temperatura de armazenamiento. Bragantia 64(2), 273-289.

González-Benito, M.E., N. Aguilar y T. Ávila. 2009. Germination and embryo rescue from Passiflora spp. seeds post-cryopreservation. CryoLetters 30(2), 142-147.

Guevara, C., J. Ospina, L. Caicedo, V. Barney y G. Coppens d'Eeckenbrugge. 2003. Criopreservación de semillas en tres especies de pasiflora. Unidad de Recursos Genéticos, CIAT, Cali; IPGRI, Roma.

Gutiérrez, M.I., D. Miranda y J.F. Cárdenas. 2011. Efecto de tratamientos pregerminativos sobre la germinación de semillas de gulupa (Passiflora edulis Sims.), granadilla (Passiflora ligularis Juss.) y cholupa (Passiflora maliformis L.). Rev. Colomb. Cienc. Hortic. 5(2), 209-219.

Hong, T.D y R.H. Ellis. 1996. A protocol to determine seed storage behavior. IPGRI Technical Bulletin No. 1. International Plant Genetic Resources Institute, Roma.

Hong, T.D., S. Linington y R.H. Ellis. 1998. Compendium of information on seed storage behaviour. Vols. I y II. Royal Botanic Gardens Kew, London.

ISTA, International Seed Testing Association 2009. International rules for seed testing, Chapter 5 Germination test. Bassersdorf, Suiza.

Júnior, M., A. São José, T. Rebouças, O. Morais y F. Dourado. 2009. Superação de dormência de maracujá-do-mato (Passiflora cincinnata Mast.). Rev. Bras. Frutic. 32(2), 584-590.
Lima, C.S.M., D.L. Betemps, Z.F.P. Tomaz, S.P. Galarça y A.R. Rufato. 2009. Germinação de sementes e crescimento de maracujá em diferentes concentrações do ácido giberélico, tempos de imersão e condições experimentais. Rev. Bras. Agroc. 15(1-4), 43-48.

Liu, S., F. Yang, J. Li, C. Zhang, H. Ji y P. Hong. 2008. Physical and chemical analysis of Passiflora seeds and seed oil from China. Int. J. Food Sci., Nutr. 59(7, 8), 706-15.

Lobo, M. 2006. Recursos genéticos y mejoramiento de frutales andinos: una visión conceptual. Rev. Corpoica Cienc. Tecnol. Agropecu. 7(2), 40-54.

Maciel, N., D. Bautista y J. Aular. 1997. Growth and development of grenadilla plants I. Morphology during the first phases of the growth cycle. Fruits 52, $11-17$.

Macguire, J.D. 1962. Speed of germination. Aid in selection and evaluation of seedling emergence and vigor. Crop Sci. 2, 176 - 177.

Manjkhola, S., U. Dhar y R.S. Rawal. 2003. Treatments to improve seed germination of Arnebia benthamii: an endangered medicinal herb of high altitude $\mathrm{Hi}$ malaya. Seed Sci. Technol. 31, 571-577.

Mendiondo, G.M y M.T. Garcia. 2009.Germination of stored and scarified seeds of Passiflora caerulea L. (Passifloraceae). Plant Biosyst. 143(2) 369 - 376.

Nakagawa, J., C. Cavariani y W.E.N. Amaral. 1991. Armazenamento de sementes de maracujá amarelo. Rev. Bras. Sem. 13(1), 77-80.

Ocampo, J. 2007. Study of the genetic diversity of genus Passiflora L. and its distribution in Colombia. Ph.D. thesis. Centre International d'Etudes Supérieures en Sciences Agronomiques - SupAgro Montpellier, Francia.

Osipi, E.A y J. Nakagawa. 2005. Efeito da temperatura na avaliação da qualidade fisiológica de Sementes do maracujá-doce (Passiflora alata Dryander). Rev. Bras. Frutic. 1(27), 179-181.

Ospina, J.A., C.L. Guevara, L.E. Caicedo y V. Barney. 2000. Effects of moisture content on Passiflora seed viability after immersion in liquid nitrogen. JIRCAS International Agriculture Series 8, 378-381.

Passos, I.R., G.V. Mato, L.M. Meletti, M.D. Scott, L.C. Bernacci y M.A. Vieira. 2004. Utilização do ácido giberélico para a quebra de dormência de sementes de Passiflora nitida Kunth germinadas in vitro. Rev. Bras. Frutic. 2(26), 380-381.

Parra, M., P.A. Rivera, R. Rodríguez y O.E. Aguilar. 2013. Acuerdo de competitividad para la cadena 
productiva de pasifloras en Colombia. Asohofrucol, CEPASS, Consejo Nacional de Pasifloras, Ministerio de Agricultura y Desarrollo Rural (MADR), Bogotá.

Ramírez, C., S. Henk, W.M. Hilhorst y E. Hodson. 2008. Viability and seed germination of Passiflora mollissima (H.B.K) Bailey according to provenance and fruit ripening stage. pp. 145-190. En: Memorias Red-Alfa Lagrotech, Unión Europea, Cartagena, Colombia.

Rao, N.K., J. Hanson, M.E. Dullo, K. Ghoh, D. Novell y M. Larinde. 2007. Manual para el manejo de semillas en bancos de germoplasma. Manuales para el manejo de bancos de germoplasma No.8. Bioversity International, Roma.

Rendón, J.S., J. Ocampo y R. Urrea. 2013. Estudio sobre polinización y biología floral en Passiflora edulis $\mathrm{f}$. edulis Sims, como base para el premejoramiento genético. Acta Agron. 62(3), 232-241.

Rosseto, C.A., R.C. Coneglian, J. Nakagawa, M.K. Shimizu y V.A. Marin. 2000. Germinação de sementes de maracujá-doce (Passiflora alata Dryand) em função de tratamento pré-germinativo. Rev. Bras. Sem. 1(22), 247-252.

Teng, Y.T. 1977. Storage of passion fruit (Passiflora edulis forma flavicarpa) seeds. The Malaysian Agric. J. 51, 118-123.

Torres, A.M. y R. Ellis. 2007. Conservación ex situ de semillas de frutos jugosos. Actual. Biol. 29(1), 88-89.

Veiga-Barbosa, L., S. Mira, M.E. González-Benito, M.M. Souza, L.M.M. Meletti y F. Pérez-García. 2013. Seed germination, desiccation tolerance and cryopreservation of Passiflora species. Seed Sci. Technol. 41, 89-97.

Wagner, J.A., R.S. Alexandre, J.R. da Silva, L. Duarte, J.O. da Costa y C.H. Bruckner. 2006. Influência do substrato na germinação e desenvolvimento inicial de plantas de maracujazeiro amarelo (Passiflora edulis Sims f. flavicarpa Deg.). Ciênc. Agrotec. 30(4), 643-647.

Westengen, O.T., S. Jeppson y L. Guarino. 2013. Global Ex-Situ Crop Diversity Conservation and the Svalbard Global Seed Vault: Assessing the Current Status. Plos One. 8(5), 1-10. 